OPEN ACCESS

Edited by:

Zhugen Yang,

Cranfield University, United Kingdom

Reviewed by:

Peiqing Liu,

Fujian Academy of Agricultural

Sciences, China

Tohid Mahmoudi,

Tabriz University of Medical

Sciences, Iran

*Correspondence:

Shijun $\mathrm{Li}$

zjumedjun@163.com

Specialty section:

This article was submitted to

Biosensors and Biomolecular

Electronics,

a section of the journal

Frontiers in Bioengineering and

Biotechnology

Received: 14 August 2021

Accepted: 26 October 2021

Published: 18 November 2021

Citation:

Yang $X$, Wang $Y$, LiU Y, Huang J,

Tan Q, Ying X, Hu Y and Li S (2021) A

Label-Based Polymer Nanoparticles

Biosensor Combined with LoopMediated Isothermal Amplification for Rapid, Sensitive, and Highly Specific Identification of Brucella abortus. Front. Bioeng. Biotechnol. 9:758564.

doi: 10.3389/fbioe.2021.758564

\section{A Label-Based Polymer Nanoparticles Biosensor Combined with Loop-Mediated Isothermal Amplification for Rapid, Sensitive, and Highly Specific Identification of Brucella abortus}

\author{
Xinggui Yang ${ }^{1,2}$, Yue Wang ${ }^{1}$, Ying Liu ${ }^{1}$, Junfei Huang ${ }^{1}$, Qinqin $\operatorname{Tan}^{1,2}$, Xia Ying ${ }^{1,2}$, Yong $\mathrm{Hu}^{2}$ \\ and Shijun $L i^{1,2 *}$ \\ ${ }^{1}$ Guizhou Provincial Center for Disease Control and Prevention, Guiyang, China, ${ }^{2}$ School of Public Health, the Key Laboratory of \\ Environmental Pollution Monitoring and Disease Control, Ministry of Education, Guizhou Medical University, Guiyang, China
}

Brucella abortus (B. abortus), an important zoonotic pathogen in Brucella spp., is the major causative agent of abortion in cattle (namely, bovine brucellosis). Currently, although the isolation and identification of the Brucella abortus were commonly accepted as the gold standard method, it cannot meet the requirements for early diagnostic strategies. Conventional PCR techniques and immunological tests can realize rapid detection of $B$. abortus, but the demands for PCR thermal cyclers and/or specific antibodies hinder their application in basic laboratories. Thus, rapid, sensitive, and specific diagnostic strategies are essential to prevent and control the spread of the bovine brucellosis. In this work, a novel detection method for the rapid identification of $B$. abortus, which uses loop-mediated isothermal amplification (LAMP) combined with a label-based polymer nanoparticles lateral flow immunoassay biosensor (LFIA), was established. One set of specific $B$. abortus-LAMP primers targeting the BruAb2_0168 gene was designed by the online LAMP primer design tool. The B. abortus-LAMP-LFIA assay was optimized and evaluated using various pathogens and whole blood samples. The optimal amplification temperature and time for $B$. abortus-LAMP-LFIA were determined to be $65^{\circ} \mathrm{C}$ and $50 \mathrm{~min}$, respectively. The $B$. abortus-LAMP-LFIA method limit of detection (LoD) was $100 \mathrm{fg}$ per reaction for pure genomic DNA of $B$. abortus. Meanwhile, the detection specificity was $100 \%$, and there was no cross-reactivity for other Brucella members and non-Brucella strains. Furthermore, the entire procedure, including the DNA preparation for whole blood samples (30 min), isothermal incubation (50 min), and LFIA detection (2-5 min), can be completed in approximately $85 \mathrm{~min}$. Thus, the B. abortus-LAMP-LFIA assay developed

\footnotetext{
Abbreviations: ATCC, American type culture collection; B. abortus, Brucella abortus; CL, control line; ddH2O, double distilled water; FAM, carboxy fluorescein; GZCDC, Guizhou Provincial Center for Disease Control and Prevention; LAMP, loopmediated isothermal amplification; LFIA, lateral flow immunoassay biosensor; LAMP-LFIA, loop-mediated isothermal amplification linked to a nanoparticle-based lateral flow immunoassay biosensor; LoD, limit of detection; MG, malachite green; nt, nucleotide; PCR, polymerase chain reaction; TL, test line.
} 
was a simple, rapid, sensitive, and reliable detection technique, which can be used as a screening and/or diagnostic tool for $B$. abortus in the field and basic laboratories.

Keywords: Brucella abortus, nanoparticles biosensor, loop-mediated isothermal amplification, BruAb2_0168 gene, blood

\section{INTRODUCTION}

Brucellosis is a worldwide major zoonotic disease caused by members of the genus Brucella, with more than half a million new cases reported annually (Hinić et al., 2008; Moeini-Zanjani et al., 2020). It can manifest as undulating fever with arthralgia, sometimes associated with chronic and severe complications (e.g., orchitis, spondylitis, and arthritis) and remains as a common cause of pyrexia of unknown origin (PUO) (Zhong et al., 2013; Daugaliyeva et al., 2018; Patra et al., 2019). Meanwhile, the symptoms of infection in animals include abortion, infertility, and decreased production. Brucella spp. are Gram-negative, facultative, and intracellular pathogens with 10 different species-specific host preferences (e.g., Brucella melitensis, Brucella abortus, Brucella suis, Brucella ovis, Brucella canis, Brucella neotomae, etc.) (Zhong et al., 2013; Daugaliyeva et al., 2018). Especially, Brucella abortus (B. abortus), an important pathogen of the genus Brucella, is a major causative agent of abortion and infertility in case of cattle population (namely, bovine brucellosis is an important infectious disease in the cattle population) (Ali et al., 2014; Islam et al., 2019; Khurana et al., 2021). Many countries have adopted different strategies to control this disease from their cattle herd. Thus, the ability to early, rapidly, and specially differentiate B. abortus is essential to control the disease.

Currently, isolation and identification of the Brucella spp. from blood culture are universally regarded as the standard diagnostic methods for laboratory examination (Patra et al., 2019). However, bacteriological isolation and identification methods (e.g., Rose Bengal plate test, serum agglutination test, and phage lysis test) are time-consuming and low sensitivity, and contain a risk of infection for laboratory personnel, so it is difficult to meet the requirements for early strategies ( $\mathrm{Li}$ et al., 2019a; Khurana et al., 2021). Thus, rapid, safe, and sensitive identification techniques are required for the detection of specific-species of Brucella spp. Presently, several rapid detection methods based on immunological tests, including enzyme-linked immunosorbent assay (ELISA) and fluorescence polarization immunoassay (FPIA) have been successfully applied for the serological diagnosis of brucellosis (Xu et al., 2020; Dong et al., 2021). Although the sensitivity of the immunological techniques is between 65 and 95\%, the low sensitivity of the acute phase, the low specificity of the antibodies, and the need for expensive reagents are major limitations of the methods (Özdemir et al., 2011; Karthik et al., 2014).

In recent years, various molecular detection techniques for the identification of Brucella spp. have been developed. Among them, polymerase chain reaction (PCR) and PCR-based assay (i.e., multiplex PCR and real-time PCR) are used as the conventional molecular detection methods in clinical examinations of brucellosis (Hinić et al., 2008; Surucuoglu et al., 2009; Kang et al., 2011). Despite these methods own outstanding analytical capabilities, the shortcomings (special apparatus' requirements, poor availability, and long detection procedure) restrict their application in the point-of-care and field laboratories (Li et al., 2019a; Yang et al., 2021). To address the shortcomings of PCR-based techniques, loop-mediated isothermal amplification (LAMP), which includes 4 core primers (F3, B3, FIP, and BIP) and/or two loop primers (LF and LB) on target sequences, was developed in 2000 by Notomi et al. (2000). LAMP technique, as a fast, reliable, simple, and sensitive isothermal detection method, has been performed to detect various pathogens (containing bacteria, viruses, fungi, and emerging/re-emerging infectious agents) (Wang et al., 2017; Kashir and Yaqinuddin, 2020; Yang et al., 2021). In previous reports, the LAMP technique has been used to detect Brucella ssp. in diagnosis assay. Unfortunately, conventional validation methods for LAMP amplicons, including hydroxy naphthol blue (HNB), SYBR Green, and agarose gel electrophoresis, are difficult to accurately distinguish specific amplification from nonspecific amplification, which can easily lead to misinterpretation of the results (Li et al., 2019a; Yang et al., 2021).

To overcome these defects, a target-specific, simple, and visual nanoparticle-based lateral flow immunoassay biosensor (LFIA) was successfully designed and applied to verify LAMP products (Li et al., 2019a; Yang et al., 2021). The detection mechanism of LFIA is that LAMP reaction amplicons, which are composed of 6carboxyfluorescein (6-FAM) and biotin, combined with dye streptavidin-coated polymer nanoparticles (SA-PNPs) to form a complex (6-FAM-biotin-SA-PNPs). Then, after the complex is captured by the rabbit anti-fluorescein antibody (anti-FITC) on the LFIA, the detection for pathogen nucleic acid is realized by the visual test line (Gong et al., 2019; Li et al., 2020). Although LAMP- and MCDA-LFIA (multiple cross displacement amplification) methods targeting Bscp31, mcr-1, IS6110, and mtp40 gene for detection of Brucella spp. (genus level) and/or other target pathogens have been established, these methods cannot accurately detect and/or identify the Brucella abortus strains (species level) (Li et al., 2019a; Li et al., 2019b; Gong et al., 2019; Yang et al., 2021). Previously, conventional PCR and LAMP assays targeting BruAb2_0168 gene were developed and applied to identify the $B$. abortus in accurate diagnosis tests of brucellosis (Hinić et al., 2008; Kang et al., 2015).

In this report, a novel LFIA detector linked to LAMP technique (LAMP-LFIA) was developed and used for the visual, simple, sensitive, and specific identification of $B$. abortus (B. abortus-LAMP-LFIA) by highly specific region on the BruAb2_0168 gene. These improvements overcome the detection complexity of traditional methods and realize the accurate identification of specific-species of Brucella spp. 
A

(1)

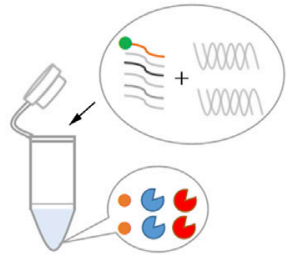

LAMP reagents
(2)

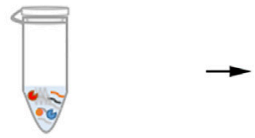

LAMP amplification $\left(65^{\circ} \mathrm{C}, 50 \mathrm{~min}\right)$
(3)

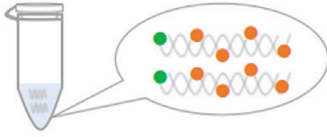

LAMP amplicons

\begin{tabular}{|c|c|c|c|c|}
\hline $\begin{array}{l}\text { 6-carboxyfluorescein } \\
\text { (6-FAM) }\end{array}$ & Biotin-14-dctp & DNA template & Bst 2.0 & Biotin/FAM-labelled \\
\hline $\begin{array}{l}\text { FAM-labelled FIP } \\
\text { primer }(B a-F I P *)\end{array}$ & A set of LAMP primers & Visual reagent & & LAMP amplicon \\
\hline
\end{tabular}

B
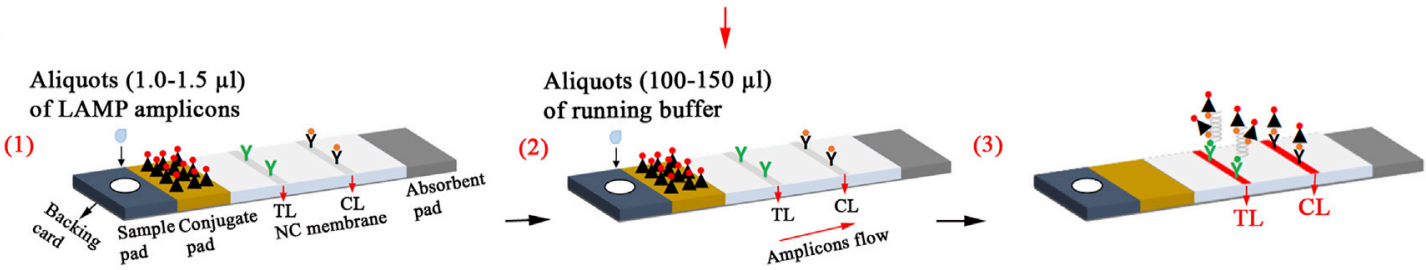

\begin{tabular}{|c|c|c|c|c|c|}
\hline $\mathbf{Y}$ & $\mathbf{Y}$ & 8 & - & $\Delta$ & $\dot{Y}$ \\
\hline Anti-FITC & $\begin{array}{l}\text { Bovine serum } \\
\text { albumin (BSA) }\end{array}$ & $\begin{array}{l}\text { Dye streptavidin-coated } \\
\text { polymer nanoparticle (SA-PNP) }\end{array}$ & $\begin{array}{l}\text { Polymer } \\
\text { nanoparticle }\end{array}$ & Streptavidin & Biotin-BSA \\
\hline
\end{tabular}

FIGURE 1 | Schematic description of the B. abortus-LAMP-LFIA assay. (A1), Preparing the reaction mixtures. (A2), B. abortus-LAMP amplification. (A3), The detectable LAMP amplicons after amplification. B. abortus-LAMP amplicons were simultaneously labeled with FAM and biotin. (B1), Aliquots (1.0-1.5 $\mu$ l) of LAMP amplicons was deposited on the sample pad of LFIA. (B2), Aliquots (100-150 $\mu$ l) of running buffer were also deposited on the sample pad of LFIA. (B3), The biotin/FAMlabeled amplification products were captured by the anti-fluorescein body (anti-FITC) fixed on the test line (TL) of the biosensor; the surplus SA-PNPs (dye streptavidin-coated polymer nanoparticle, crimson red) were captured by the biotin-BSA (biotinylated bovine serum albumin) fixed on the control line (CL) of the biosensor, which demonstrated the working condition of the biosensor. 6-FAM, 6-carboxyfluorescein; B. abortus, Brucella abortus; LAMP, loop-mediated isothermal amplification; LFIA, lateral flow immunoassay biosensor.

(namely Brucella abortus). In B. abortus-LAMP-LFIA system, a unique-region of BruAb2_0168 gene was amplified in the reaction mixture, and results were indicated using LFIA. The optimal reaction conditions and feasibility of the $B$. abortus-LAMP-LFIA assay were confirmed by using DNA from pure cultures and whole blood samples.

\section{MATERIALS AND METHODS}

\section{Ethical Statement}

The study was approved by the Human Ethics Committee of the Guizhou Provincial Center for Disease Control and Prevention and complied with the Declaration of Helsinki. All data/isolates were analyzed anonymously.

\section{Reagents and Apparatus}

Universal DNA isothermal amplification kits and visual malachite green (MG) were provided by Bei-Jing HaiTaiZhengYuan. Co., Ltd. (Beijing, China). Bacterial genomic DNA extraction kits were purchased from Takara Biomedical Technology Co., Ltd. (Beijing, China). Biotin-14dctp $(0.1 \mathrm{mM})$ was provided by Tian-Jin Huidexin Technology
Development Co., Ltd. (Tianjin, China). These materials including backing card, sample pad, conjugate pad, nitrocellulose membrane (NC), and absorbent pad were provided by Jie-Yi Biotechnology. Co., Ltd. (Shanghai, China). Biotinylated bovine serum albumin (biotin-BSA) and anti-FITC were purchased from Abcam. Co., Ltd. (Shanghai, China). Dye (crimson red) streptavidin-coated polymer nanoparticles (SAPNP) $\left(129 \mathrm{~nm}, 10 \mathrm{mg} \mathrm{ml}^{-1}, 100 \mathrm{mM}\right.$ borate, $\mathrm{pH} 8.5$ with $0.1 \%$ BSA, $0.05 \%$ Tween 20 , and $10 \mathrm{mM}$ EDTA) were obtained from Bangs Laboratories, INC. (Indiana, United States).

\section{Preparation of the Nanoparticle-Based Biosensor}

Nanoparticle-based biosensors $(60 \times 4 \mathrm{~mm})$ were designed based on a previous publication in our study (Wang et al., 2018). Briefly, the sample pad, conjugate pad, NC membrane, and absorbent pad were attached to a plastic adhesive backing card. Then, the capture reagents, containing anti-FITC $(0.15 \mathrm{mg} / \mathrm{ml})$ and biotin-BSA $(2.5 \mathrm{mg} / \mathrm{ml})$ in $0.01 \mathrm{M}$ phosphate buffered saline (PBS, PH 7.4), were immobilized on the NC membrane. Thus, there were two bands, including control line (CL) conjugated with biotin-BSA and test line (TL) conjugated with anti-FITC, which 


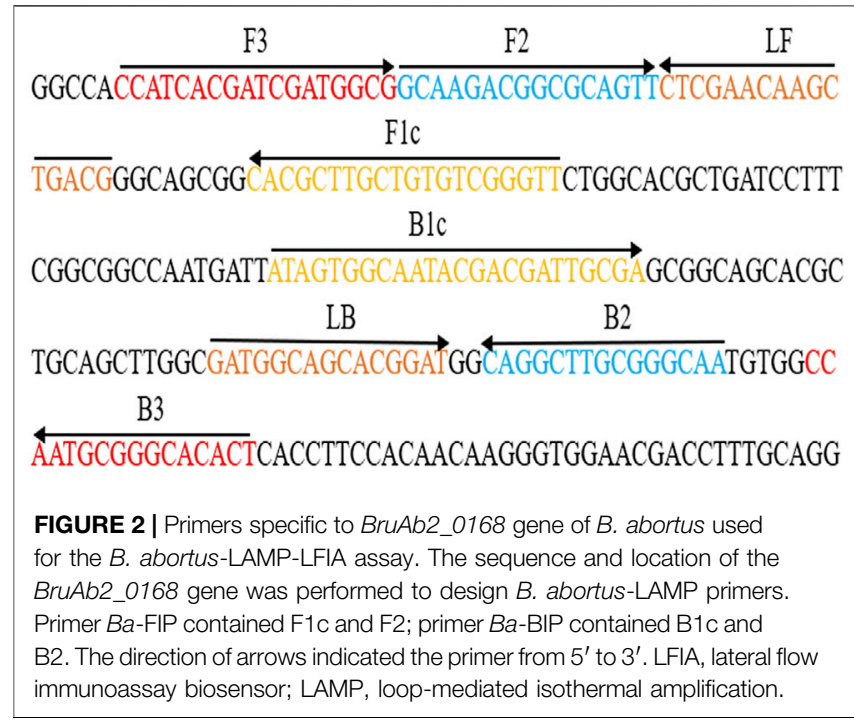

were separated by $5 \mathrm{~mm}$. SA-PNPs $\left(129 \mathrm{~nm}, 10 \mathrm{mg} \mathrm{ml}^{-1}\right.$, $100 \mathrm{mM}$ borate, $\mathrm{pH} 8.5$ with $0.1 \%$ BSA, $0.05 \%$ Tween 20 , and $10 \mathrm{mM}$ EDTA) in $0.01 \mathrm{M}$ PBS (PH 7.4) were collected in the conjugate pad. The assembled cards were cut into $4-\mathrm{mm}$ wide strips (Deli No. 8012). The assembled biosensors were packaged in a plastic box containing a desiccant gel and stored in a dry and dark place (room temperature). According to our design, the LFIAs were timely manufactured by Tian-Jin HuiDeXin Biotech. Co., Ltd. (Tianjin, China). A schematic description of the $B$. abortus-LAMP-LFIA assay is displayed in Figure 1. An aliquot (1.0-1.5 $\mu \mathrm{l}$ ) of LAMP amplicons was deposited on the sample pad of LFIA, and then an aliquot $(100-150 \mu \mathrm{l})$ of running buffer is also deposited on the sample pad of LFIA in our study.

\section{Loop-Mediated Isothermal Amplification Primers Design and Screening}

After sequence alignment and screening using the BLASTn (Basic Local Alignment Search Tool), six primers ( $B a$-FIP, $B a$-BIP, $B a$ $\mathrm{LF}, B a-\mathrm{LB}, B a-\mathrm{F} 3$ and $B a-\mathrm{B} 3)$ for the $B r u A b 2 \_0168$ gene (GenBank accession no. AE017224.1) was designed via online website (primerexplorer.jp/lampv5e/index.html). 6-FAM was labeled on the $5^{\prime}$ end of the $B a$-FIP primer. The primer information (i.e., sequence, length, and modification) is shown in Figure 2 and Table 1. All primers (HPLC purification grade) used in our study were synthesized and purified by TianyiHuiyuan Biotech Co., Ltd. (Beijing, China). More than 4 sets of LAMP primer targeting BruAb2_0168 gene were designed and synthesized, and they were used to screen the optimal primer set by observing specific-amplification, cross-reactivity, and reaction speed in the current report.

\section{Bacterial Strains and DNA Extraction}

A total number of 16 Brucella strains, including $B$. abortus (reference strain 544, isolated strains, vaccine strain A19), $B$. melitensis (reference strain $16 \mathrm{M}$, isolated strains, vaccine strains M5 and M28), B. suis (reference strain 1330S, isolated strains, vaccine strain S2), and $B$. canis (isolated strain), and 7 nonBrucella isolates were used in this report (Table 2). Then, DNA templates for 23 bacterial strains were prepared using the bacterial genomic DNA extraction kits, and stored at $-20^{\circ} \mathrm{C}$. The genomic DNA for B. abortus 544 was tested at the $260 / 280$ wavelengths using an ultraviolet spectrophotometer, and serial dilutions ( $1 \mathrm{ng}, 100 \mathrm{pg}, 10 \mathrm{pg}, 1 \mathrm{pg}, 100 \mathrm{fg}, 10 \mathrm{fg}$, and $1 \mathrm{fg}$ per microliter) were prepared for primer sets screening, confirmation test, reaction temperature and time optimization, sensitivity, and specificity analysis.

\section{Processing the Whole Blood Samples}

A total of 86 whole blood samples, which were suspected from bovine brucellosis, were collected from different regions of Guizhou province, China. All blood samples were divided equally into two parts (Part I and Part II). Whole blood samples (Part I) were cultured using the BACTEC FX system (Becton-Dickinson, Sparks, MD), incubated for 6 weeks and subcultured weekly (Sagi et al., 2017). Briefly, the blood samples (approximately $3 \mathrm{ml}$ ) collected from cattle that were aseptically inoculated into a two-phase culture flask (BIOVD, Zhengzhou, Henan, China) to cultivate and isolate Brucella strains. Postincubation at the conditions of $37^{\circ} \mathrm{C}$ with $5 \% \mathrm{CO}_{2}$ for $3-5$ days (or more 3-5 days cultivation for blind passage), the bacteria strain was streaked on blood agar plate and Brucella agar plate for pure cultivation. Suspected Brucella strains (genus level) were identified based on conventional biochemical tests, Gram staining and serum agglutination tests (Susceptible and Variant, 1990). Subsequent B. abortus strains (species level) were further identified using phage lysis tests in Brucella isolates according to a previous publication (Susceptible and Variant, 1990). The blood samples (Part II) were subjected to B. abortus-PCR and B. abortus-LAMP-LFIA assays by using the

\section{TABLE 1 | The primers used in the B. abortus-LAMP-LFIA assay.}

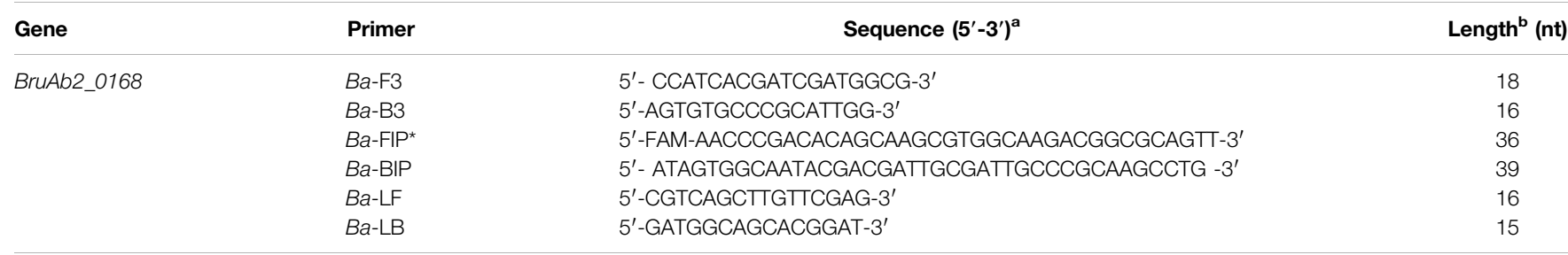

${ }^{a}$ FAM, 6-Carboxyfluorescein.

${ }^{b}$ nt, nucleotide. 
TABLE 2 | The information of bacterial strains in this study.

\begin{tabular}{|c|c|c|c|}
\hline Bacteria & $\begin{array}{l}\text { Strain no. (source } \\
\text { of strains) }\end{array}$ & No. of strains & LAMP-LFIA result ${ }^{\mathbf{b}}$ \\
\hline \multicolumn{4}{|l|}{ Brucella species } \\
\hline B. abortus & A19 (GZCDC) & 1 & $P$ \\
\hline B. abortus & 2038 (GZCDC) & 1 & $P$ \\
\hline B. abortus & Isolated strain (GZCDC) & 4 & $P$ \\
\hline B. melitensis & 16M (ATCC 23456) & 1 & $N$ \\
\hline B. melitensis & Isolated strains (GZCDC) & 2 & $N$ \\
\hline B. suis & 1330S (ATCC 23444) & 1 & $N$ \\
\hline B. suis & S2 (GZCDC) & 1 & $N$ \\
\hline B. suis & Isolated strain (GZCDC) & 1 & $N$ \\
\hline B. canis & Isolated strain (GZCDC) & 1 & $\mathrm{~N}$ \\
\hline \multicolumn{4}{|l|}{ Non-Brucella species } \\
\hline Klebsiella pneumoniae & Isolated strain (GZCDC) & 1 & $N$ \\
\hline Pseudomonas aeruginosa & Isolated strain (GZCDC) & 1 & $N$ \\
\hline Streptococcus pneumoniae & Isolated strain (GZCDC) & 1 & N \\
\hline Total & & 23 & - \\
\hline
\end{tabular}

${ }^{a}$ ATCC, american type culture collection; GZCDC, guizhou provincial center for disease control and prevention.

${ }^{b} P$, positive; $N$, negative.

protocol of QIAamp to directly extract DNA templates from these samples $(500 \mu \mathrm{l})$ (Li et al., 2019a).

\section{The Standard Loop-Mediated Isothermal Amplification Reaction}

The availability of optimal LAMP primer for BruAb2_0168 gene was confirmed using the standard LAMP reaction with the follow-up test ( $\mathrm{Li}$ et al., 2019a). The reaction system $(25 \mu \mathrm{l})$ of LAMP assay containing the following: $12.5 \mu \mathrm{l} 2 \times$ reaction buffer, $1 \mu \mathrm{l} 2.0$ Bst DNA polymerase, $1.6 \mu \mathrm{M}$ each of FIP $^{\star}$ and BIP, $0.8 \mu \mathrm{M}$ each of LF and LB, $0.4 \mu \mathrm{M}$ each of F3 and B3, $1 \mu \mathrm{l}$ of biotin-14-dCTP, $1 \mu \mathrm{l}$ MG indicator, DNA templates ( $1 \mu \mathrm{l}$ of pure culture and $4 \mu \mathrm{l}$ of samples), and double distilled water $\left(\mathrm{ddH}_{2} \mathrm{O}\right)$ were added to $25 \mu \mathrm{l}$. The reaction tubes were incubated at $63^{\circ} \mathrm{C}$ for $60 \mathrm{~min}$ and then it was terminated at $85^{\circ} \mathrm{C}$ for $5 \mathrm{~min}$. Finally, the LAMP amplicons were verified using nanoparticle-based biosensor, MG reagents, and real-time turbidimeter $L A-500$ (Eiken Chemical Co., Ltd. Japan).

\section{Optimization of Reaction Temperature and Time}

Then, the optimal temperature of the B. abortus-LAMP-LFIA assay was confirmed by setting the different reaction temperature $\left(63-70^{\circ} \mathrm{C}\right.$, intervals $\left.1^{\circ} \mathrm{C}\right)$, and the template DNA $(10 \mathrm{pg} / \mu \mathrm{l})$ of $B$. abortus 544 was employed in this study. The assay was performed according to the standard LAMP assay and monitored using the turbidimeter. In addition, the threshold value (turbidity) was 0.1 , and a turbidity of $>0.1$ was considered as positive amplification (Li et al., 2019a).

Moreover, the effect of different times (10-60 min, with $10 \mathrm{~min}$ intervals) on $B$. abortus-LAMP-LFIA assay was evaluated, and the amplicons were detected with biosensors. When MG reagent was added to the reaction mixtures, the color of reaction tubes for LAMP positive amplification changed from dark blue to blue, while the negative control and blank control were light blue or colorless. In the experiment, $1 \mu \mathrm{l}$ genomic DNA of Listeria monocytogenes and/ or Mycobacterium tuberculosis was used as negative control (NC) and $1 \mu \mathrm{l}$ of $\mathrm{ddH}_{2} \mathrm{O}$ was used as blank control (BC).

\section{Sensitivity and Specificity of the $B$. abortus-LAMP-LFIA Assay}

The sensitivity of the $B$. abortus-LAMP-LFIA assay was confirmed using serial dilutions $(1 \mathrm{ng} / \mu \mathrm{l}-1 \mathrm{fg} / \mu \mathrm{l})$ of the template DNA of $B$. abortus 544, and the test was performed using a defined number of replicates (usually 20 per dilution). The limit of detection (LoD) of the LAMP-LFIA was defined as the lowest concentration of genomic DNA that, when detected by serial dilutions, resulted in the detection of B. abortus in $\geq 95 \%$ of the assays conducted in the current research (Chakravorty et al., 2017). The results of sensitivity assays were reported by LFIAs, MG indicator, and turbidimeter, and all tests were independently implemented in multiple replicates.

In order to evaluate the specificity of B. abortus-LAMP-LFIA assay, genomic DNA of 23 bacterial strains was detected 


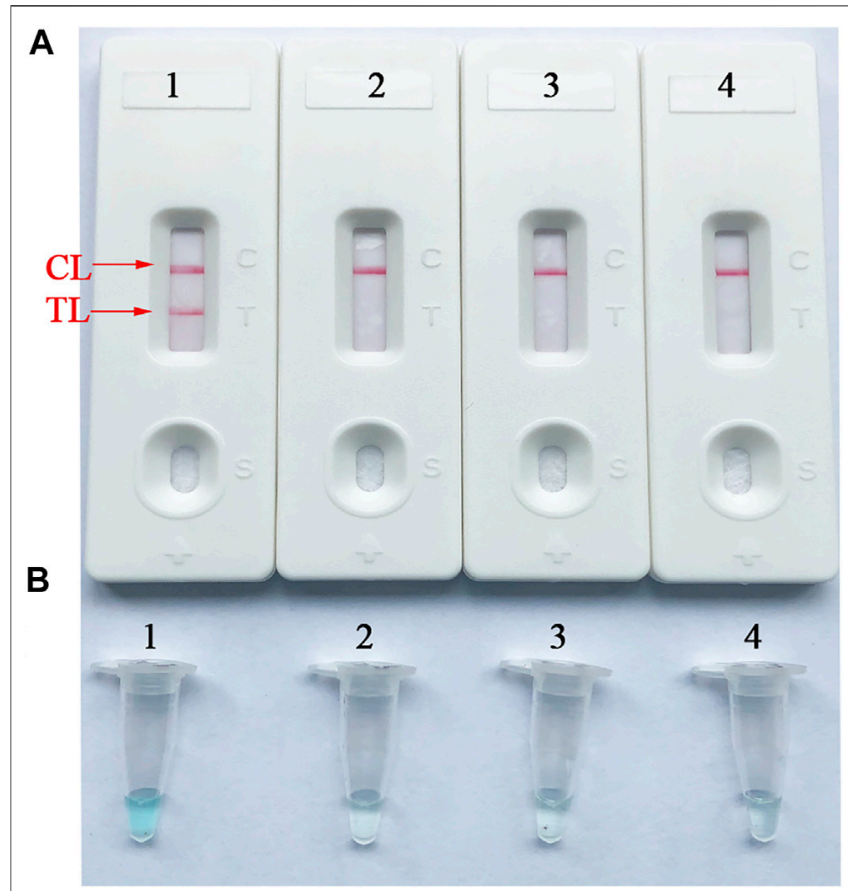

FIGURE 3 | Verification and analysis of B. abortus-LAMP-LFIA amplicons. (A) The LFIA strips used for rapid detection of $B$. abortus-LAMPLFIA products. (B) Color change for $B$. abortus-LAMP-LFIA tubes. Strip/tube 1: positive reaction of $B$. abortus-LAMP products for the $B$. abortus 544; strip/tube 2: negative control (Listeria monocytogenes); strip/tube 3: negative control (Mycobacterium tuberculosis); strip/tube 4: blank control $\left(\mathrm{ddH}_{2} \mathrm{O}\right)$. LAMP, loop-mediated isothermal amplification; LFIA, lateral flow immunoassay biosensor; $\mathrm{TL}$, test line; $\mathrm{CL}$, control line; $\mathrm{ddH}_{2} \mathrm{O}$, double distilled water.

according to the optimal amplification temperature and time (Table 2). The assay results were verified using LFIAs.

\section{Applicability of the B. abortus-LAMP-LFIA Assay to Whole Blood Samples}

In order to evaluate the practicability of $B$. abortus-LAMP-LFIA assay for B. abortus detection, 86 samples were tested by culturebiotechnical methods (i.e., Gram staining, biochemical tests, serum agglutination tests, and phage lysis tests), B. abortus-PCR, and $B$. abortus-LAMP-LFIA assay. According to the abovementioned extraction steps of genomic DNA from whole blood samples, $4 \mu \mathrm{l}$ of template DNA was used for B. abortus-PCR and B. abortus-LAMPLFIA tests. B. abortus-PCR tests were performed with reference to previous publications (Hinić et al., 2008), and the reaction mixtures $(25 \mu \mathrm{l})$ contained the following: $12.5 \mu \mathrm{l} 2 \times$ Taq Master Mix (CoWin Biosciences Co., Ltd. Beijing, China), $0.2 \mu \mathrm{M} B a$-F (5' ${ }^{\prime}$ TCG-CAT-

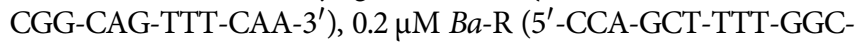
CTT-TTC-C-3'), $4 \mu \mathrm{l}$ of samples DNA, and $\mathrm{ddH}_{2} \mathrm{O}$ was added to $25 \mu$. The reactions were carried out using an automated thermal cycler (Thermo Fisher Scientific Co., Ltd. Beijing, China). The reaction mixtures were denatured at $94^{\circ} \mathrm{C}$ for $2 \mathrm{~min}$, and 35 reaction cycles were conducted. The cycles consisted of denaturation at $94^{\circ} \mathrm{C}(30 \mathrm{~s})$, annealing at $59^{\circ} \mathrm{C}(30 \mathrm{~s})$, and primer extension at $72^{\circ} \mathrm{C}(30 \mathrm{~s})$. The final extension time was set for $2 \mathrm{~min}$. The PCR products were visualized in a $2.0 \%$ agarose gel with GelRed staining under UV light (BioRad, United States). The B. abortus-LAMP-LFIA assay was applied according to optimal reaction conditions.

\section{Statistical Analysis}

In this report, the results of 86 whole blood samples tested by culturebiotechnical methods (as the standard method) were used as the standard, and they were further analyzed and calculated for sensitivity (\%), specificity (\%), positive predictive value (PPV\%), and negative predictive value (NPV\%) of B. abortus-PCR and B. abortus-LAMPLFIA methods using SPSS software (ver. 26.0; IBM, United States).

\section{RESULTS}

\section{Confirmation and Validation of $B$. abortus-LAMP Amplicons}

To confirm the availability of an optimal primer set screened from more than 4 primer sets, B. abortus-LAMP tests were performed using the genomic DNA $(10 \mathrm{pg} / \mu \mathrm{l})$ of $B$. abortus 544 . The CL (control line) and TL (test line) were red for positive amplification, while only the CL line was red for negative and blank control (Figure 3A). Meanwhile, the positive amplification tubes changed from dark blue to blue, while the negative tubes were colorless (Figure 3B).

\section{Optimal Reaction Temperature and Time for B. abortus-LAMP-LFIA Assay}

To obtain the optimal reaction temperature for the B. abortus-LAMPLFIA experiment, the optimization test was performed by setting a series of amplification temperatures $\left(60-67^{\circ} \mathrm{C}\right.$, with $1^{\circ} \mathrm{C}$ interval). Among the eight kinetics graphs generated, the $B$. abortus-LAMPLFIA assay exhibited higher amplification efficiency when the test temperature ranged from 64 to $66^{\circ} \mathrm{C}$ (Figure 4).

Different incubation times (ranging from 10 to $60 \mathrm{~min}$, with 10 -min intervals) were applied to verify the optimal reaction time for the B. abortus-LAMP-LFIA assay. Time optimization tests demonstrated that the biosensors could detect the genomic DNA of $B$. abortus 544 at a minimum concentration of $100 \mathrm{fg} / \mu \mathrm{l}$ when the reaction time was $50-60 \mathrm{~min}$ (Table 3 ). Thus, a reaction temperature of $65^{\circ} \mathrm{C}$ and an amplification time of $50 \mathrm{~min}$ were used as the optimal conditions for the rest of the B. abortusLAMP-LFIA assays in the current study.

\section{Detection Sensitivity of $B$. abortus-LAMP-LFIA Assay}

The sensitivity of $B$. abortus-LAMP-LFIA method was confirmed by repeated detection for serial dilutions of genomic DNA of $B$. abortus 544 in our study. The B. abortus-LAMP-LFIA assay LoDs were $100 \mathrm{fg}$ of template DNA per microliter. TL and CL lines (red) could be observed on LFIA, indicating positive amplification for BruAb2_0168 gene (Figure 5A). In particular, LFIA verification results for LAMP amplicons were consistent with MG visual indicators (Figure 5B) and real-time turbidity (Figure 5C). 

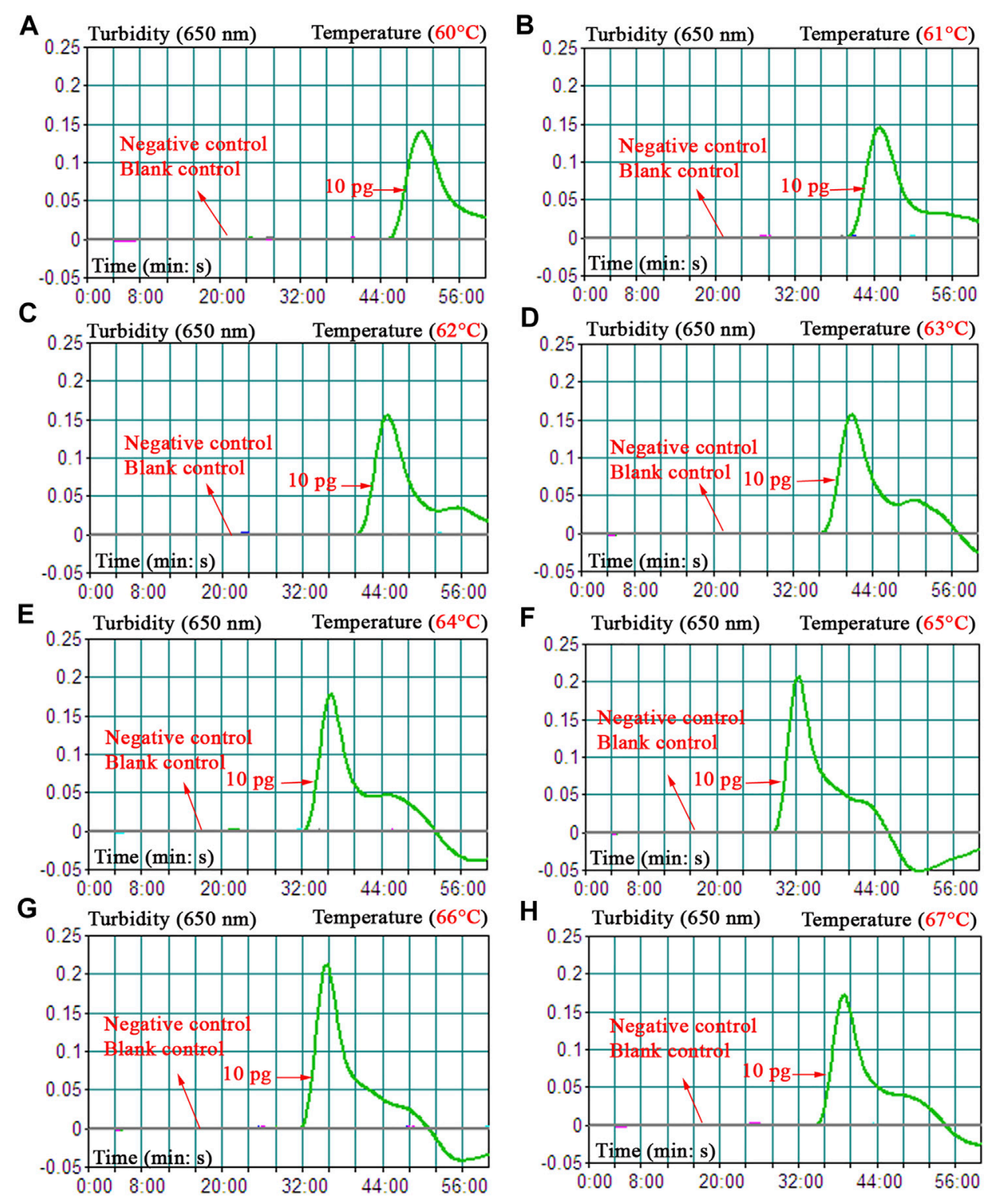

F Turbidity $(650 \mathrm{~nm}) \quad$ Temperature $\left(65^{\circ} \mathrm{C}\right)$
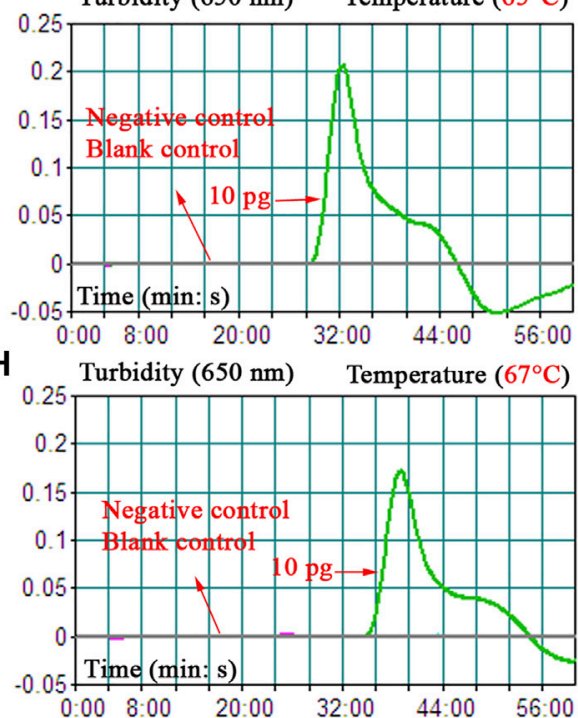

FIGURE 4 | Optimization of amplification temperature for the $B$. abortus-LAMP-LFIA assay. The standard B. abortus-LAMP assays were monitored by real-time turbidimetry, and the corresponding information was marked in the drawings. This threshold value was 0.1 , and a turbidity $>0.1$ was judged as a positive reaction. $A$ total of eight kinetic graphs $(\mathbf{A}-\mathbf{H})$ were generated at various temperatures $\left(60-67^{\circ} \mathrm{C}, 1^{\circ} \mathrm{C}\right.$ intervals) with template DNA at the level of 10 pg/ $\mathrm{Hl}$. The drawings from $\mathbf{( E - G )}$ showed higher amplification efficiency. LAMP, loop-mediated isothermal amplification; LFIA, lateral flow immunoassay biosensor; TL, test line; CL, control line; $\mathrm{dd}_{2} \mathrm{O}$, double distilled water; $\mathrm{BC}$, blank control.

\section{Detection Specificity of $B$. abortus-LAMP-LFIA Assay}

The detection specificity of LAMP-LFIA was evaluated using genomic DNA extracted from 23 bacterial strains (i.e., Brucella species/strains and non-Brucella strains) (Table 2). The analysis specificity of B. abortus-LAMP-LFIA was $100 \%$ in our study. The $B$. abortus-LAMP-LFIA method can specifically detect representative strains of $B$. abortus (isolates, reference, and vaccine strains), but other bacterial pathogens (other Brucella members and non-Brucella strains) cannot be detected (Figure 6).

\section{Practical Evaluation of the $B$. abortus-LAMP-LFIA Assay for Whole Blood Samples}

A total of 86 whole blood samples were tested by conventional culturebiotechnical methods, B. abortus-PCR, and B. abortus-LAMP-LFIA 
TABLE 3 | The optimization of amplification time for the B. abortus-LAMPLFIA assay.

\begin{tabular}{lcccccccc} 
Time/min & \multicolumn{7}{c}{ Serial dilutions of genomic DNA (B. abortus 544) } \\
\cline { 2 - 8 } & $\mathbf{1} \mathbf{~ n g}$ & $\mathbf{1 0 0} \mathbf{~ p g}$ & $\mathbf{1 0} \mathbf{~ p g}$ & $\mathbf{1} \mathbf{~ p g}$ & $\mathbf{1 0 0} \mathbf{f g}^{\mathbf{a}}$ & $\mathbf{1 0} \mathbf{f g}$ & $\mathbf{1} \mathbf{f g}$ & $\mathbf{B C}^{\mathbf{b}}$ \\
\hline 10 & - & - & - & - & - & - & - & - \\
20 & + & + & - & - & - & - & - & - \\
30 & + & + & + & + & - & - & - & - \\
40 & + & + & + & + & $+/-$ & - & - & - \\
50 & + & + & + & + & + & - & - & - \\
60 & + & + & + & + & + & - & - & -
\end{tabular}

+ positive amplification; -, negative amplification.

${ }^{a}+/-$, weak positive amplification.

${ }^{b} B C$, blank control.

assay as described above. Then, the practicability of $B$. abortus-LAMPLFIA assay was further evaluated by comparing with the results of culture-biotechnical methods and B. abortus-PCR assay. In the whole blood sample detection results, 9 samples were tested as positive, and 77 samples were negative by the culture-biotechnical methods. Nine were detected as positive and 77 were negative by B. abortus-LAMPLFIA assay. However, only 7 samples were examined as positive, and 79 samples were negative by $B$. abortus-PCR method. Both the sensitivity and specificity of B. abortus-LAMP-LFIA was $100 \%$ (based on the standard results of culture-biotechnical methods) (Table 4). These results show that the B. abortus-LAMP-LFIA assay developed in the current study was a valuable diagnostic tool to detect whole blood samples.

\section{DISCUSSION}

Currently, although the isolation and identification (namely culture-biotechnical methods) of the Brucella spp. were commonly accepted as the gold standard method, these

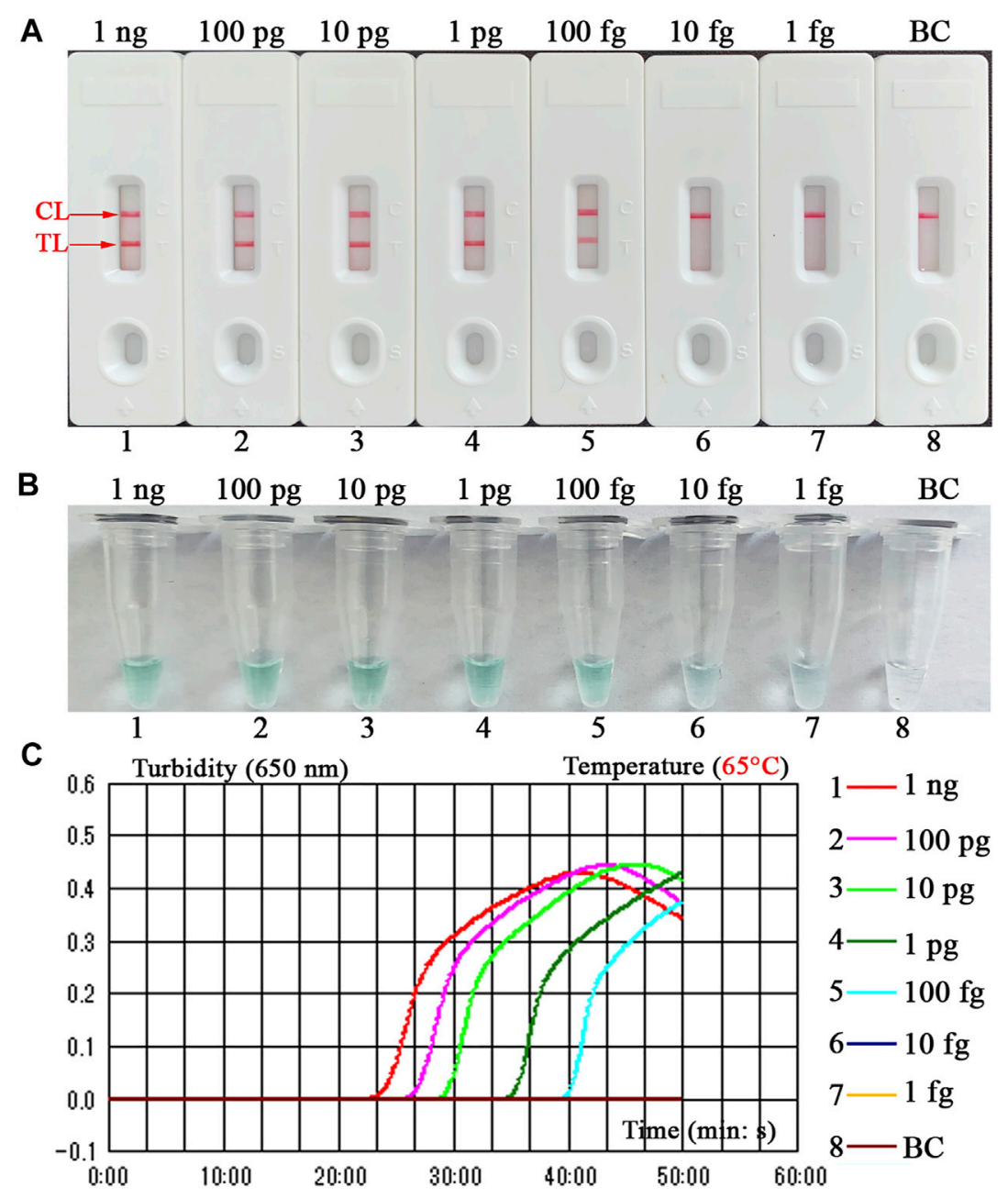

FIGURE 5 | Detection sensitivity for the B. abortus-LAMP-LFIA assay. The experiments were performed according to the optimal reaction condition, and serial dilutions (1 ng, 100 pg, 10 pg, 1 pg, 100 fg, 10 fg, and $1 \mathrm{fg}$ per microliter) of target DNA template were tested. Three validation tools, containing LFIA strips (A), MG reagents (B), and real-time turbidity (C), were used to verify the B. abortus-LAMP amplification products. Strips (A)/tubes (B)/curves (C) 1-7 correspond to DNA template of $B$. abortus 544 from $1 \mathrm{ng} / \mu \mathrm{ll}$ to $1 \mathrm{fg} / \mu \mathrm{l}$, strip/tube/curve 8: blank control $\left(\mathrm{ddH}_{2} \mathrm{O}\right)$. MG, malachite green; LAMP, loop-mediated isothermal amplification; $\mathrm{LFIA}$, lateral flow immunoassay biosensor; $\mathrm{ddH}_{2} \mathrm{O}$, double distilled water; $\mathrm{TL}$, test line; $\mathrm{CL}$, control line. All tests were independently implemented in multiple replicates. 


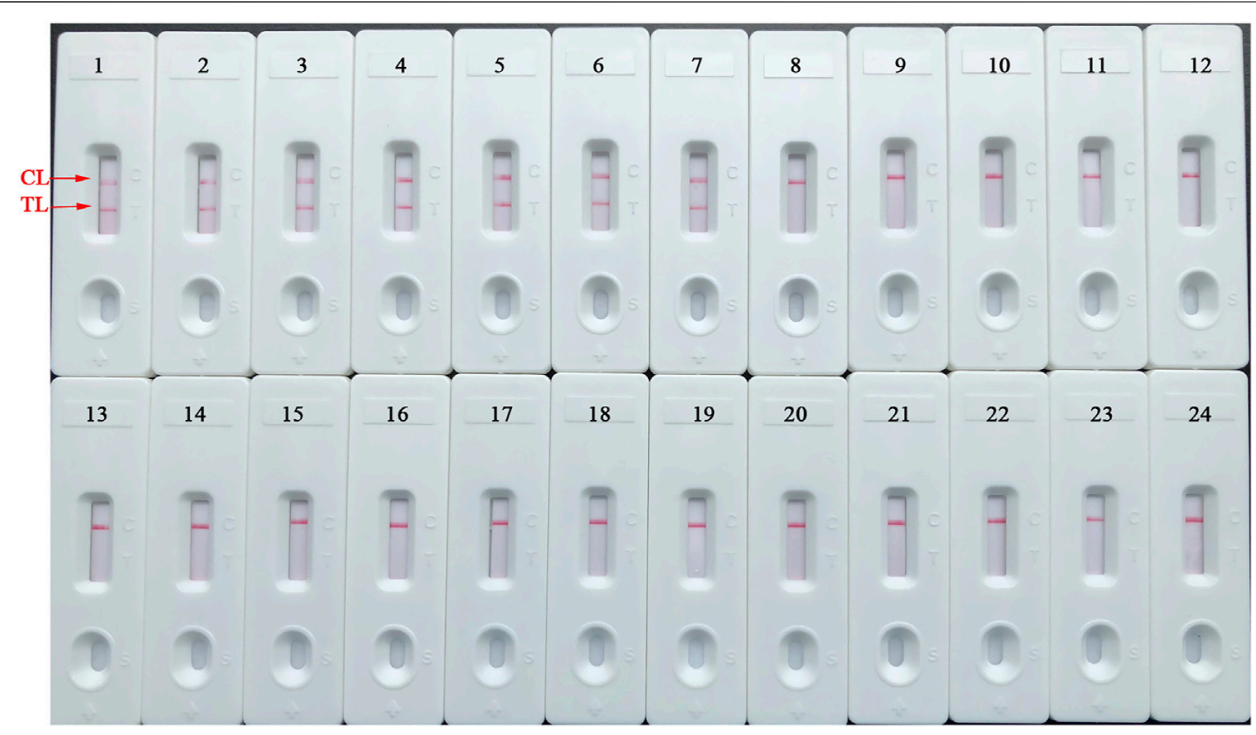

FIGURE 6 | Detection specificity of $B$. abortus-LAMP-LFIA assay for various pathogens. The $B$. abortus-LAMP tests were performed using template DNA of different pathogens, and the amplicons were verified by LFIA strips. Strips 1-3, B. abortus 544 (ATCC 23448), B. abortus A19, and B. abortus 2038; strips 4-7, B. abortus (isolates); strips 8-10, B. melitensis 16M (ATCC 23456), B. melitensis M28, and B. melitensis M5; strips 11-12, B. melitensis (isolates); strips 13-16, B. suis 1330 S (ATCC 23444), B. suis S2, B. suis (isolate), and B. canis (isolate); strips 17-23, Mycobacterium tuberculosis, Mycobacterium bovis, Listeria monocytogenes, Salmonella spp., Klebsiella pneumoniae, Pseudomonas aeruginosa, and Streptococcus pneumoniae; strip 24, blank control (ddH ${ }_{2} \mathrm{O}$ ). LAMP, loopmediated isothermal amplification; LFIA, lateral flow immunoassay biosensor; $\mathrm{TL}$, test line; $\mathrm{CL}$, control line; $\mathrm{dd}_{2} \mathrm{O}$, double distilled water; $\mathrm{BC}$, blank control; $\mathrm{ATCC}$, American Type Culture Collection.

TABLE 4 | Comparison of culture-biotechnical, B. abortus-PCR and B. abortus-LAMP-LFIA assay for the detection of B. abortus in whole blood samples.

\begin{tabular}{|c|c|c|c|c|c|c|}
\hline \multirow[t]{2}{*}{ Detection methods ${ }^{a}$} & \multicolumn{2}{|c|}{ Culture-biotechnical } & \multirow[t]{2}{*}{ Sensitivity (\%) } & \multirow[t]{2}{*}{ Specificity (\%) } & \multirow[t]{2}{*}{ PPV $^{b}(\%)$} & \multirow[t]{2}{*}{ NPV $^{c}(\%)$} \\
\hline & Positive ( $\mathbf{N}=9$ ) & Negative $(\mathbf{N}=77)$ & & & & \\
\hline \multicolumn{7}{|l|}{ B. abortus-PCR } \\
\hline Positive & 7 & 0 & 77.78 & 100 & 100 & 97.47 \\
\hline Negative & 2 & 77 & & & & \\
\hline \multicolumn{7}{|l|}{ B. abortus-LAMP-LFIA } \\
\hline Positive & 9 & 0 & 100 & 100 & 100 & 100 \\
\hline Negative & 0 & 77 & & & & \\
\hline
\end{tabular}

${ }^{a} \mathrm{~B}$. abortus, Brucella abortus; PCR, polymerase chain reaction; LAMP, loop-mediated isothermal amplification; LFIA, lateral flow immunoassay biosensor.

${ }^{b} P P V$, positive predictive value; $P P V=$ (true positive/true positive + false positive) * 100.

${ }^{c} N P V$, negative predictive value; NPV = (true negative/true negative + false negative) * 100.

deficiencies (e.g., time-consuming, complicated operation steps and the risk of infection to laboratory personnel) cannot meet the requirements for early strategies (Schwarz et al., 2017; Li et al., 2019a). However, conventional PCR techniques (single PCR, multiplex PCR, and real-time PCR) and immunological tests (ELISA and FPIA) can realize more rapid detection of Brucella spp. than the culture-biotechnical methods, but the demands for PCR thermal cyclers and/or specific antibodies hinder their development in basic laboratories (Surucuoglu et al., 2009; Kang et al., 2011; Xu et al., 2020; Dong et al., 2021). Thus, it is extremely necessary to develop newly diagnostic methods that can meet the above-mentioned requirements.

LAMP, as a low-cost, fast, simple, and efficient nucleic acid amplification technique, seems to be more satisfactory. Presently, LAMP- and LAMP-based assay have been applied in the detection of various pathogens (containing viruses, bacteria, and fungi) (Sharma et al., 2016; Gomes et al., 2020). However, conventional validation tools for LAMP amplicons, including visual indicators, agarose gel electrophoresis, and real-time turbidimeter, are difficult to achieve simple, rapid, and accurate detection of target pathogens (Li et al., 2019a; Yang et al., 2021). A label-based polymer nanoparticle biosensor was developed and applied to specifically verify LAMP amplification products by labeling special primer ( $\mathrm{Ba}$-FIP primer was labeled 6FAM) (Li et al., 2019b; Yang et al., 2021). The test results can be verified visually (approximately $2 \mathrm{~min}$ ) by observing the color of the TL line (red and/or colorless) on the LFIA strips. Although LAMP- and MCDA-LFIA methods targeting Bscp31 gene for detection of Brucella spp. (genus level) have been established in previous publications, these two assays cannot accurately identify 
the B. abortus strains (species level) (Li et al., 2019b; Li et al., 2019a). Meanwhile, conventional LAMP techniques targeting the BruAb2_0168 gene have achieved rapid detection of B. abortus and demonstrated reliable specificity and sensitivity, the shortcomings of frequently used confirmation methods for LAMP amplicons are still unavoidable in a previous work (Karthik et al., 2014). Thus, the nanoparticle biosensor combined with the LAMP technique targeting BruAb2_0168 gene (B. abortus-LAMP-LFIA) has been successfully established and performed to achieve rapid and accurate detection of $B$. abortus in the current report.

A set of unique LAMP primers specifically recognized 8 regions on the BruAb2_0168 sequence, thus showing high selectivity for the diagnosis of $B$. abortus strains (Figure 2). In order to confirm the optimal reaction conditions of LAMP-LFIA to obtain more efficient amplification, different reaction temperatures $\left(60-67^{\circ} \mathrm{C}, 1^{\circ} \mathrm{C}\right.$ interval $)$ and times (10-60 $\mathrm{min}, 10-\mathrm{min}$ intervals) were carried out in our study. When the reaction condition was at $65^{\circ} \mathrm{C}$ for $50 \mathrm{~min}, B$. abortus-LAMP-LFIA assay showed relatively stable amplification (Figure 4 and Table 3). Meanwhile, the specificity of B. abortus-LAMP-LFIA assay was $100 \%$, which can not only detect all representative $B$. abortus strains (i.e., reference strains, vaccine strain, and isolates), but also exclude other Brucella species (including $B$. melitensis, B. suis, and B. canis) and non-Brucella strains (Figure 6).

In addition, $B$. abortus-LAMP-LFIA showed excellent detection sensitivity and could be detected when the genomic DNA concentration of B. abortus 544 was $100 \mathrm{fg}$ per microliter (Figure 5). The sensitivity of the novel nanoparticle-based biosensors combined with LAMP assay was tenfold higher than that of PCR assay (Alamian et al., 2019; Moeini-Zanjani et al., 2020). In follow-up studies, after appropriately extending the amplification time $(70-80 \mathrm{~min})$, we found that B. abortusLAMP-LFIA assay can detect the genomic DNA concentration of B. abortus 544 at about $80 \mathrm{fg}$ per reaction (data not shown). In addition to using LFIAs to verify LAMP products, we also added MG amplification indicator to the reaction mixture in the experiment, and the results of amplification can be preliminarily judged with the naked eye. As expected, compared with the verification results of LFIAs, positive and negative amplifications do not seem to be clearly distinguished by visual indicators (e.g., the color change between $100 \mathrm{fg}$ and $10 \mathrm{fg}$ reaction tubes). However, the LAMP reaction is prone to aerosol contamination, so the whole experiment was performed in different laboratories (e.g., sample preparation, premixed reaction mixture, LAMP amplification, and LAMP amplicons verification).

In this study, in order to evaluate the practicality of B. abortusLAMP-LFIA in practical examination, a total of 86 whole blood samples collected from suspected bovine brucellosis were tested using culture-biotechnical, conventional B. abortus-PCR, and B. abortus-LAMP-LFIA methods. The accurate diagnosis efficiency of $B$. abortus-LAMP-LFIA was consistent with the culturebiotechnical methods (Table 4). In addition, ROC analysis (AUC 1.000, the data not shown) based on the sensitivity and specificity of $B$. abortus-LAMP-LFIA was further carried out in our study using the $\mathrm{R}$ package (ver. 4. 0. 5). Interestingly, we found that two samples, which were tested as positive by $B$. abortus-LAMP-LFIA and culture-biotechnical, were detected as negative by $B$. abortus-PCR assay. The reasons for the different results are as follows: (i) the concentration of template DNA in the whole blood sample is low and cannot reach the minimum detection limit of B. abortus-PCR methods (PCR assay LoDs are usually 1-100 pg per microliter for genomic DNA) (MoeiniZanjani et al., 2020). (ii) This phenomenon may be caused by false-positive amplification of B. abortus-LAMP-LFIA, and the non-specific amplification of conventional LAMP assay was occasionally reported in a previous publication; however, the possibility of false-positives for $B$. abortus-LAMP-LFIA is extremely low, due to these novel strategies were performed (including the specific labeling of primers, the design and application of LFIA biosensors, and the confirmation of conventional culture-biotechnical methods) (Li et al., 2019a; Yang et al., 2021). Meanwhile, 86 blood samples were also examined by the Brucella spp.-LAMP-LFIA assay established in our previous experiments (Li et al., 2019a), and the positive results were consistent with $B$. abortus-LAMP-LFIA (data not shown). These data indicate that although the B. abortus-LAMPLFIA method is as sensitive as the culture-biotechnical methods for the detection of practical whole blood samples in the current experiment, $B$. abortus-LAMP-LFIA is simpler, faster, safer, and more applicable than the culture-biotechnical methods.

Moreover, compared to other verification tools for LAMP amplicons (including color indicator, real-time turbidity, and agarose gel electrophoresis), nanoparticle-based LFIA is more convenient (amplification products and running buffer are added to the sample pad), visual (the results are determined by directly observing the color changes of CL and TL line), and highly specific (the $B a$-FIP primer is specifically labeled). Meanwhile, the total cost of a single $B$. abortus-LAMP-LFIA reaction is about 5 USD, including LAMP isothermal reagents (approximately 1.5 USD), LFIA biosensor (approximately 2.5 USD), DNA extraction reagents (approximately 0.2 USD), MG indicator reagents (approximately $0.3 \mathrm{USD}$ ), and other reagents and/or materials (approximately 0.5 USD).

\section{CONCLUSION}

In this report, a simple, visual, and reliable nanoparticle-based LFIA, which can eliminate the use of special equipment and simplify the detection procedure, was newly designed and applied in the current report. The $B$. abortus-LAMP-LFIA assay ( $B$. abortus-LAMP combined with a nanoparticle-based LFIA) targeting the BruAb2_0168 gene was successfully established and performed, and the technique showed excellent sensitivity and specificity for the detection of bacterial strains (including reference strains, vaccine strains, and isolates) and whole blood samples. Hence, the B. abortus-LAMP-LFIA test developed was a simple, rapid, sensitive, and reliable detection technique, which can be used as a screening and/or diagnostic tool for B. abortus in the field and basic laboratories. 


\section{DATA AVAILABILITY STATEMENT}

The raw data supporting the conclusion of this article will be made available by the authors, without undue reservation.

\section{AUTHOR CONTRIBUTIONS}

X.Y. and S.L. conceived and designed this study. S.L. supervised the study. X.Y., S.L., Y.W., Y.L., J.H., Q.T., X.Y., and Y.H. conducted the experiments. X.Y., S.L., Y.W., and Y.L. analyzed

\section{REFERENCES}

Alamian, S., Zahraei Salehi, T., Aghaiypour Kolyani, K., Esmaelizad, M., and Etemadi, A. (2019). Development of New Modified Simple Polymerase Chain Reaction and Real-Time Polymerase Chain Reaction for the Identification of Iranian Brucella Abortus Strains. Arch. Razi Inst. 74, 235-241. doi:10.22092/ ARI.2018.122128.1218

Ali, S., Ali, Q., Melzer, F., Khan, I., Akhter, S., Neubauer, H., et al. (2014). Isolation and Identification of Bovine Brucella Isolates from Pakistan by Biochemical Tests and PCR. Trop. Anim. Health Prod. 46, 73-78. doi:10.1007/S11250-0130448-6

Chakravorty, S., Simmons, A. M., Rowneki, M., Parmar, H., Cao, Y., Ryan, J., et al. (2017). The New Xpert MTB/RIF Ultra: Improving Detection of Mycobacterium tuberculosis and Resistance to Rifampin in an Assay Suitable for point-of-care Testing. MBio 8, 1-12. doi:10.1128/mBio.00812-17

Daugaliyeva, A., Sultanov, A., Usserbayev, B., Baramova, S., Modesto, P., Adambayeva, A., et al. (2018). Genotyping of Brucella Melitensis and Brucella Abortus Strains in Kazakhstan Using MLVA-15. Infect. Genet. Evol. 58, 135-144. doi:10.1016/J.MEEGID.2017.12.022

Dong, S.-B., Xiao, D., Liu, J.-Y., Bi, H.-M., Zheng, Z.-R., Wang, L.-D., et al. (2021). Fluorescence Polarization Assay Improves the Rapid Detection of Human Brucellosis in China. Infect. Dis. Poverty 10, 1-6. doi:10.1186/s40249-02100834-3

Gomes, Y., Caterino-De-Araujo, A., Campos, K., Gonçalves, M. G., Leite, A. C., Lima, M. A., et al. (2020). Loop-Mediated Isothermal Amplification (LAMP) Assay for Rapid and Accurate Confirmatory Diagnosis of HTLV-1/2 Infection. Viruses 12, 1-15. doi:10.3390/v12090981

Gong, L., Liu, E., Che, J., Li, J., Liu, X., Xu, H., et al. (2019). Multiple Cross Displacement Amplification Coupled with Gold Nanoparticles-Based Lateral Flow Biosensor for Detection of the Mobilized Colistin Resistance Gene Mcr-1. Front. Cell. Infect. Microbiol. 9, 1-9. doi:10.3389/fcimb.2019.00226

Hinić, V., Brodard, I., Thomann, A., Cvetnić, Ž., Makaya, P. V., Frey, J., et al. (2008). Novel Identification and Differentiation of Brucella Melitensis, B. Abortus, B. Suis, B. ovis, B. canis, and B. Neotomae Suitable for Both Conventional and Real-Time PCR Systems. J. Microbiol. Methods 75, 375-378. doi:10.1016/j.mimet.2008.07.002

Islam, M. S., Garofolo, G., Sacchini, L., Dainty, A. C., Khatun, M. M., Saha, S., et al. (2019). First Isolation, Identification and Genetic Characterization ofBrucella Abortusbiovar 3 from Dairy Cattle in Bangladesh. Vet. Med. Sci. 5, 556-562. doi:10.1002/vms3.193

Kang, S.-I., Her, M., Kim, J.-Y., Lee, J. J., Lee, K., Sung, S.-R., et al. (2015). Rapid and Specific Identification of Brucella Abortus Using the Loop-Mediated Isothermal Amplification (LAMP) Assay. Comp. Immunol. Microbiol. Infect. Dis. 40, 1-6. doi:10.1016/j.cimid.2015.03.001

Kang, S.-I., Her, M., Kim, J. W., Kim, J.-Y., Ko, K. Y., Ha, Y.-M., et al. (2011). Advanced Multiplex PCR Assay for Differentiation of Brucella Species. Appl. Environ. Microbiol. 77, 6726-6728. doi:10.1128/AEM.00581-11

Karthik, K., Rathore, R., Thomas, P., Arun, T. R., Viswas, K. N., Agarwal, R. K., et al. (2014). Loop-mediated Isothermal Amplification (LAMP) Test for Specific and Rapid Detection ofBrucella Abortusin Cattle. Vet. Q. 34, 174-179. doi:10.1080/01652176.2014.966172 the data. S.L., Y.W., Y.L., J.H., and Y.H. contributed the reagents and analysis tools. S.L., Y.L., Y.W., J.H., and Y.H. contributed the materials. X.Y. performed the software. X.Y. drafted the manuscript. S.L. revised the manuscript.

\section{FUNDING}

This study was funded by grants from Science and Technology Department of Guizhou Province (No. (2016)4021 and (2018) 5606).

Kashir, J., and Yaqinuddin, A. (2020). Loop Mediated Isothermal Amplification (LAMP) Assays as a Rapid Diagnostic for COVID-19. Med. Hypotheses 141, 109786. doi:10.1016/J.MEHY.2020.109786

Khurana, S. K., Sehrawat, A., Tiwari, R., Prasad, M., Gulati, B., Shabbir, M. Z., et al. (2021). Bovine Brucellosis - a Comprehensive Review. Vet. Q. 41, 61-88. doi:10.1080/01652176.2020.1868616

Li, S., Jiang, W., Huang, J., Liu, Y., Ren, L., Zhuang, L., et al. (2020). Highly Sensitive and Specific Diagnosis of COVID-19 by Reverse Transcription Multiple Cross-Displacement Amplification-Labelled Nanoparticles Biosensor. Eur. Respir. J. 56, 2002060. doi:10.1183/ 13993003.02060-2020

Li, S., Liu, Y., Wang, Y., Chen, H., Liu, C., and Wang, Y. (2019a). Lateral Flow Biosensor Combined with Loop-Mediated Isothermal Amplification for Simple, Rapid, Sensitive, and Reliable Detection of Brucella Spp. Idr 12, 2343-2353. doi:10.2147/IDR.S211644

Li, S., Liu, Y., Wang, Y., Wang, M., Liu, C., and Wang, Y. (2019b). Rapid Detection of Brucella Spp. And Elimination of Carryover Using Multiple Cross Displacement Amplification Coupled with Nanoparticles-Based Lateral Flow Biosensor. Front. Cell. Infect. Microbiol. 9, 1-11. doi:10.3389/fcimb.2019.00078

Moeini-Zanjani, A., Pournajaf, A., Ferdosi-Shahandashti, E., Gholami, M., Masjedian, F., Khafri, S., et al. (2020). Comparison of Loop-Mediated Isothermal Amplification and Conventional PCR Tests for Diagnosis of Common Brucella Species. BMC Res. Notes 13, 8-12. doi:10.1186/s13104020-05377-8

Notomi, T., Okayama, H., Masubuchi, H., Yonekawa, T., Watanabe, K., Amino, N., et al. (2000). Loop-mediated Isothermal Amplification of DNA. Nucleic Acids Res. 28, 63e-63. doi:10.1093/NAR/28.12.E63

Özdemir, M., Feyzioğlu, B., Kurtoğlu, M. G., Doğan, M., Dağı, H. T., Yüksekkaya, Ş., et al. (2011). A Comparison of Immuncapture Agglutination and ELISA Methods in Serological Diagnosis of Brucellosis. Int. J. Med. Sci. 8, 428-432. doi:10.7150/ijms.8.428

Patra, S., Tellapragada, C., Vandana, K. E., and Mukhopadhyay, C. (2019). Diagnostic Utility of In-house Loop-mediated Isothermal Amplification and real-timePCRtargetingvirBgene for Direct Detection ofBrucella Melitensisfrom Clinical Specimens. J. Appl. Microbiol. 127, 230-236. doi:10.1111/jam.14260

Sagi, M., Nesher, L., and Yagupsky, P. (2017). The Bactec FX Blood Culture System Detects brucella Melitensis Bacteremia in Adult Patients within the Routine 1week Incubation Period. J. Clin. Microbiol. 55, 942-946. doi:10.1128/ JCM.02320-16

Schwarz, N. G., Loderstaedt, U., Hahn, A., Hinz, R., Zautner, A. E., Eibach, D., et al. (2017). Microbiological Laboratory Diagnostics of Neglected Zoonotic Diseases (NZDs). Acta Tropica 165, 40-65. doi:10.1016/ J.ACTATROPICA.2015.09.003

Sharma, M., Sharma, K., Sharma, A., Gupta, N., and Rajwanshi, A. (2016). Loopmediated Isothermal Amplification (LAMP) Assay for Speedy Diagnosis of Tubercular Lymphadenitis: The Multi-Targeted 60-minute Approach. Tuberculosis 100, 114-117. doi:10.1016/j.tube.2016.07.015

Surucuoglu, S., El, S., Ural, S., Gazi, H., Kurutepe, S., Taskiran, P., et al. (2009). Evaluation of Real-Time PCR Method for Rapid Diagnosis of Brucellosis with Different Clinical Manifestations. Pol. J. Microbiol. 58, 15-19.

Susceptible, S., and Variant, N. (1990). Isolation, Identification. J. Clin. Microbiol. $28,1057-1059$ 
Wang, Y., Li, H., Wang, Y., Xu, H., Xu, J., and Ye, C. (20182017). Antarctic Thermolabile Uracil-DNA-Glycosylase-Supplemented Multiple Cross Displacement Amplification Using a Label-Based Nanoparticle Lateral Flow Biosensor for the Simultaneous Detection of Nucleic Acid Sequences and Elimination of Carryover Contamination. Nano Res. 11, 2632-2647. doi:10.1007/S12274-017-1893-Z

Wang, Y., Li, H., Wang, Y., Zhang, L., Xu, J., and Ye, C. (2017). Loopmediated Isothermal Amplification Label-Based Gold Nanoparticles Lateral Flow Biosensor for Detection of Enterococcus faecalis and Staphylococcus aureus. Front. Microbiol. 8, 1. doi:10.3389/ fmicb.2017.00192

Xu, N., Wang, W., Chen, F., Li, W., and Wang, G. (2020). ELISA Is superior to Bacterial Culture and Agglutination Test in the Diagnosis of Brucellosis in an Endemic Area in China. BMC Infect. Dis. 20, 1-7. doi:10.1186/s12879-0194729-1

Yang, X., Huang, J., Chen, X., Xiao, Z., Wang, X., Chen, Y., et al. (2021). Rapid and Visual Differentiation of Mycobacterium tuberculosis from the Mycobacterium tuberculosis Complex Using Multiplex LoopMediated Isothermal Amplification Coupled with a NanoparticleBased Lateral Flow Biosensor. Front. Microbiol. 12, 1-15. doi:10.3389/ fmicb.2021.708658
Zhong, Z., Yu, S., Wang, X., Dong, S., Xu, J., Wang, Y., et al. (2013). Human Brucellosis in the People's Republic of China during 2005-2010. Int. J. Infect. Dis. 17, e289-e292. doi:10.1016/J.IJID.2012.12.030

Conflict of Interest: The authors declare that the research was conducted in the absence of any commercial or financial relationships that could be construed as a potential conflict of interest.

Publisher's Note: All claims expressed in this article are solely those of the authors and do not necessarily represent those of their affiliated organizations, or those of the publisher, the editors, and the reviewers. Any product that may be evaluated in this article, or claim that may be made by its manufacturer, is not guaranteed or endorsed by the publisher.

Copyright (C) 2021 Yang, Wang, Liu, Huang, Tan, Ying, Hu and Li. This is an openaccess article distributed under the terms of the Creative Commons Attribution License (CC BY). The use, distribution or reproduction in other forums is permitted, provided the original author(s) and the copyright owner(s) are credited and that the original publication in this journal is cited, in accordance with accepted academic practice. No use, distribution or reproduction is permitted which does not comply with these terms. 